International Journal of Computer Networks \& Communications (IJCNC) Vol.10, No.1, January 2018

\title{
Quad Tree Based Static Multi Hop Leach ENERgy EFficient Routing Protocol: A NOVEL APPROACH
}

\author{
${ }^{1}$ Abdulmalek Maresh Hasan Ali and ${ }^{2}$ Hanumanthappa .J. \\ ${ }^{1}$ Research Scholar, Department of CS\&E, VTU, Belgaum, Karnataka, India. \\ ${ }^{2}$ DoS in CS, University of Mysuru, Manasagangotri, Mysuru, Karnataka, India.
}

\begin{abstract}
This research work propounds a simple graph theory semblance Divide and Conquer Quad tree based Multi-hop Static Leach (DCQMS-Leach) energy efficient routing protocol for wireless sensor networks. The pivotal theme of this research work is to demonstrate how divide and conquer plays a pivotal role in a multi-hop static leach energy efficient routing protocol. This research work motivates, enforces, reckons the DCQMS-Leach energy efficient routing protocol in wireless sensor networks using Mat lab simulator. This research work also computes the performance concepts of DCQMS-Leach routing protocol using various performance metrics such as Packet Drop Rate (PDR), Throughput, and End to End Delay (EED) by comparing and contrasting alive nodes with number of nodes, number of each packets sent to the cluster heads with rounds, number of cluster heads with rounds, number of packets forwarded to the base station with rounds and finally dead nodes with number of rounds. In order to curtail energy consumption this research work proffers a routing methodology such as DCQMS-Leach in energy efficient wireless sensor routing protocol. The recommended DCQMS-Leach overcomes the in adequacies of all other different leach protocols suggested by the previous researchers.
\end{abstract}

\section{KEYWORDS}

Divide and Conquer $(D \& C)$, Divide and Conquer Quad tree based Multi hop Static Leach (DCQMSLeach), Energy Efficient Routing Protocol (EERP), Wireless Sensor Networks (WSNs), Energy Efficient WSNs (EEWSNs).

\section{Prologue}

\subsection{Introduction To Wireless Sensor Networks And Divide And Conquer TECHNIQUE}

WSNs are networks of devices with restrained resources which are used for environmental, military, automation and home applications [6]. In the past few years wsns have increased interest in the various applications such as fault tolerance, clustering techniques, energy efficient protocols, agriculture field, body area networks (bans), under water wsns (uwwsns), medical applications such as cancer treatment, caesarean, tubectomy, vasectomy etc. Currently ameliorates in mini authorization and low power design have led to the development of smallsized battery operated sensors which are mainly used to discern the various conditions such as sound and temperature. Sensors are pivotally equipped with data processing and communication capabilities. In wsn each sensor hop mainly consists of an onboard radio which is vitally maneuvered to forward a sequence of data to a collection of communicating parties $[1,2,3,4]$. The wsn consists of a gargantuan number of tiny and low cost sensor hops powered by small rechargeable batteries equipped with different kinds of sensing devices. In this research work a vital issue to be considered from the communication point of view is, routing. Routing is a demanding job in a divide and conquer based energy efficient wsns [22]. It has already been

DOI: 10.5121/ijenc.2018.10105 
confirmed that leach protocol in energy efficient wsn is one of the algorithms for forwarding packets by contracting energy consumption of nodes [6][9][15]. But the routing methodology adopted in leach routing protocol still results in considerable energy consumption of hop for sending and receiving of data [6],[10],[37]. The DCQMS-Leach protocol [41][42] is made up of heterogeneous kinds of sensor nodes which are broadly divided into static clusters before applying DCQMS-Leach routing protocol algorithm [41,42]. Several researchers have addressed different kinds of techniques to curtail energy efficiency consumption in wsns. It has also been confirmed that many routing protocols have been maneuvered in wsns, to diminish energy consumption due to long haul transmission, by proper arrangement of hops into different areas called clusters.

Prof.Dr. Hanumanthappa .J [15...20][23...24][37...39] has compared and constituted a mathematical modeling and the comparison and dissimilitude between the performance issues of T-SEP and E-SEP energy efficient clustering protocols. He has compared and contrasted the performance issues of T-SEP and E-SEP performance metrics such as throughput, number of an alive nodes, number of dead nodes and network life time etc. They have also proved and confirmed that T-SEP is better than E-SEP in wsn when different transmission rate and phases of heterogeneity etc are applied. He is also proposing a quad tree based static multi hop energy efficient wsn routing protocols using graph theoretic approach. He has also proposed performance and evaluation of a novel energy efficient multipath routing algorithms in wsn [40]. He has also investigated how energy efficient multi hop and single hop routing protocol plays a vital role in diminishing energy consumption in eewsns [1..39].

W.Heinzelman, A.Chandrakasan, H.Balakrishnan [11,12,13] have developed LEACH (Low Energy Adaptive Clustering Hierarchy) an architecture for remote micro sensor networks, which combines the ideas of energy efficient cluster based routing and media access together with an application specific data aggregation to achieve good performance, in terms of system life time, latency and application perceived quality. They also designed and implemented LEACH protocol architecture for wireless micro sensor networks that achieves a low energy dissipation and latency without sacrificing application specific quality. One of the first hierarchical routing protocol in wsns is LEACH. In LEACH protocol the dynamic clustering technique adopts to a node which selects itself to become a cluster head by sending some probability and broadcast status into an entire network [6],[18].

Sze-yao Ni and D.Estrin have proved that clustering technique plays an important role in wireless sensor networks. The Clustering algorithm such as Low Energy Adaptive Clustering Hierarchy $(\mathrm{LEACH})$, Hybrid Energy Efficient Distributed (HEED) Clustering technique and power efficient gathering in Sensor Information Systems (PEGASIS) are all energy efficient routing protocols which are mainly used to stretch the life time of battery.

Mhatre and Rosenberg [27] have shown a cost based comparative study of homogeneous and heterogeneous clustered wsns (hcwsns). They proposed a technique to estimate the optimal distribution among different types of sensors but again this result is hard to use if the heterogeneity is due to the operation of network. They have also conducted research on Multi-hop routing within each cluster called M-LEACH (Mobile Leach). One of the short comings of the methodology is that only powerful nodes can become cluster-heads and that M-LEACH is vindicable under many assumptions and only when the population of the node is very large.

Georgios Smaragdakis,Ibrahim Matta,Azer Bestavros[15...19] have proposed SEP (Stable Election Protocol) for wsns. They have also rectified that SEP yields longer stability region for higher values of extra energy brought by more powerful nodes. In their research work they found that behavior of sensor networks become unstable once the first node dies, especially in the presence of node heterogeneity. Duerte Melo and Liu examined the performance and energy 
consumption of wsns in a field where there are two kinds of sensors. They have also estimated that the optimal percentage of powerful nodes in the field but this result is very difficult to use when heterogeneity is a type of operation of sensor network and not a choice of optimal setting.

Hence taking into account all the above research paper results we are proposing a more powerful and a simpler novel approach to diminish energy consumption in wireless sensor networks such as DCQMS-Leach routing protocol which is presented here. Thus the crux of this research work rests on discovering the energy performance by reducing energy consumption by minimizing dead nodes, increasing alive nodes and by abbreviating energy in different levels of wsns. The advantage of our newly propounded concept is divide and conquer based quad-tree concept. Our newly proposed technique has the worst case time complexity $\mathrm{O}\left(\mathrm{n}^{4}\right)$, best case time complexity $\mathrm{O}\left(\mathrm{n}^{1}\right)$ and an average case time complexity $\mathrm{O}\left(\mathrm{n}^{2}\right)$ where $\mathrm{n}$ is the number of quadrants required to split a wsn region. However our method works based on the assumption that each quadrant which consists of at least one base station (bs) $[5,6,7,8,9,10]$. This paper is organized into five sections. The section 2 describes the network model of divide and conquer based quad tree multi hop static leach protocol. The proposed methodology is set out in section 3, the simulation results and discussions are dealt with in section 4 and finally the section 5 concludes an epilogue.

\section{NETWORK MODEL}

\subsection{Graph Theoretic DCQMS-Leach Routing Protocol.}

In this research work the wireless sensor network model is elaborated. We know this from DCQMS-Leach in ewsns work on heterogeneous network. The heterogeneous network consists of $\mathrm{T}_{\mathrm{N}}$ total number of nodes which are randomly distributed across $\mathrm{H}$ x J jurisdiction. Each cluster contains $\mathrm{N}_{\mathrm{CN}}$ number of cluster nodes. The distribution of each $\mathrm{N}_{\mathrm{CN}}$ is random where the value of $\mathrm{CN}$ can vary from 1 to $\mathrm{h}$. The value of $\mathrm{h}$ is 4 . The main jurisdiction is divided into sub jurisdictions. The sub jurisdictions are also called as clusters. The creation of each cluster can be assumed as rectangle, square or both according to the network design requirement. As we know the cluster nodes (cns) are always transmitting data to the base station bs). The fig 1 shows the delineation of DCQMS-Leach energy efficient routing protocol. The different kinds of CH's forward compressed data to the base station in a wsn. The process of creating a cluster in energy efficient wsn (eewsn) is generally done by taking into account the various types of constraints such as proximity between the sensors calculated through radio frequency signal that they emit. The selection of the hop to act as a Cluster Head $(\mathrm{CH})$ or a simple energy saving hop is one of the toughest problem in eewns. To solve this problem in eewsns this research work considers power level signal, transmission schedules, localization of nodes and function of a network. Clusters also support pricing of forwarding data to the sink hop, decreasing the energy power consumption in the devices, facilitating the gathering of sensed data, increasing the routing process execution and allowing scalability. One of the techniques of sensor network in eewsns using graph theory concept is $\mathrm{G}=(\mathrm{V}, \mathrm{E})$ where $\mathrm{G}$ is wsn graph, the $\mathrm{V}$ indicates Vertices of a eewsns and $\mathrm{E}$ specifies edges of eewsns. In eewsn two types of networks are used on the basis of energy level such as homogenous and heterogeneous. The homogenous energy efficient network is one which supports node maintaining same energy level where as heterogeneous network supports the nodes with dissimilar energy levels. This mathematical modeling of DCQMS-Leach protocol always support heterogeneous network. The DCQMS-Leach protocol splits the energy levels of wsn protocols into two different types such as advanced nodes and normal nodes. The nodes maintain maximum energy level are called advanced nodes $\left(\mathrm{n}_{\mathrm{adv}}\right)$ and nodes with minimum level of energy are called normal nodes $\left(\mathrm{n}_{\mathrm{nrm}}\right)$. The actual energy percentage of advanced nodes is $\mathrm{j}$. Always each advanced node $\left(\mathrm{n}_{\text {adv }}\right)$ specifies $\mathrm{h}$ times of higher energy level than $\mathrm{n}_{\mathrm{nrm}}$. 
Normal nodes

BS

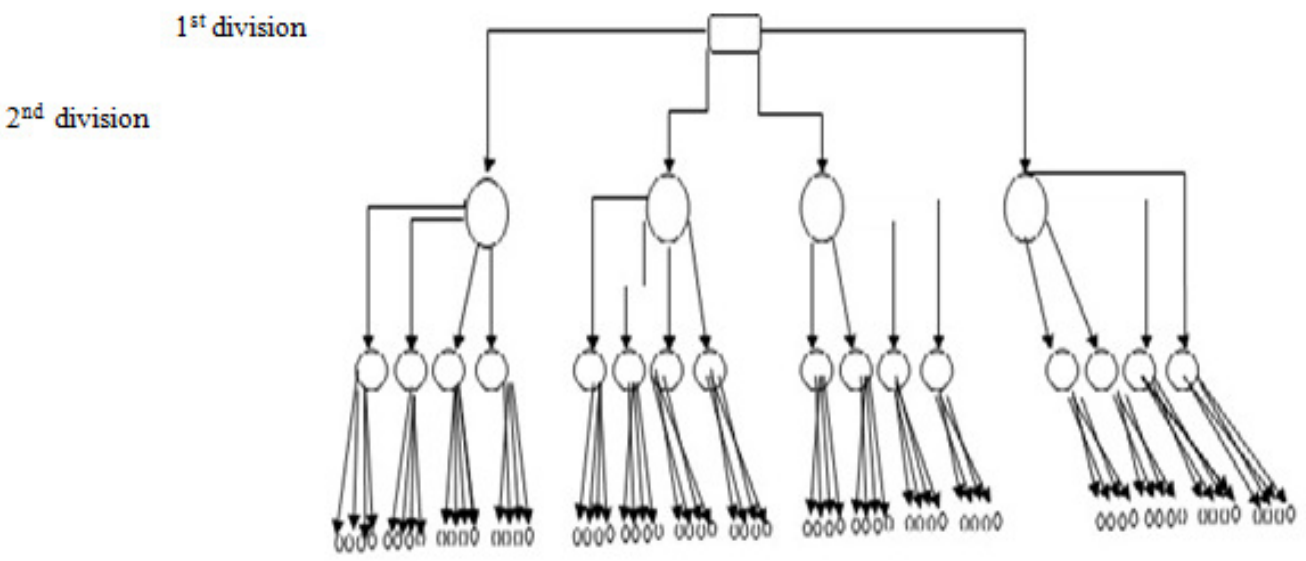

Fig 1. Specification of DCQMS-Leach Energy Efficient Routing Protocol.

\subsection{Graph TheOretic SPECification Of Cluster NodeS}

By using graph theoretic approach the cluster nodes are specified using the equation- (i).

According to the graph theoretic approach one of the idea to create a sensor network is $G=(V, E)$ where the vertices $\mathrm{V}$ specify the sensors and the set of links $\mathrm{E}$ specify the connection between vertices if they are specified within a given jurisdiction. According to the graph theoretic approach a cluster is considered as any subset of nodes such as $\mathrm{C} \subseteq \mathrm{V}, \mathrm{y} \in \mathrm{V}$ is a cluster head and $\mathrm{G}_{\mathrm{c}}$ $=\left(\mathrm{C}, \mathrm{E}_{\mathrm{c}}\right)$ is a kind of cluster graph.

$$
\mathbf{E} \mathbf{c}=\{(\mathbf{u}, \mathbf{v}) \mid \mathbf{u}, \mathbf{v} \in \mathbf{C} \wedge(u, v) \in \mathbf{E}\}
$$

When cluster graph $G_{c}$ is connected then the cluster is called as a connected graph. $d_{c}(u, v)$ is the shortest path inside a cluster. The cluster radius is the maximal distance between y and any other node $\mathrm{v} \in C$.

$$
\max _{\mathrm{v}} \in \mathbf{C d}_{\mathrm{c}}(\mathbf{y}, \mathbf{v})
$$

The energy computation of each cluster group is equal to $\mathrm{E}_{\mathrm{cl}}$. By using two levels of energy equation the cluster energy is calculated using equation (iv)

$$
\mathbf{E}_{\mathrm{cl}}=\mathbf{N}_{\mathrm{cl}} \mathbf{E}_{0}(\mathbf{l}-\mathbf{j})+\mathbf{N}_{\mathrm{cl}} \mathbf{j} \mathrm{E}_{0}(\mathbf{1}+\mathbf{h})
$$

Where $\mathrm{E}_{0}$ :-initial energy of a normal node, $(1-\mathrm{j}) \mathrm{Ncl}$ :sum of quantity of normal nodes, $\mathrm{N}_{\mathrm{cl}}$ $\mathrm{j}$ :Number of advanced nodes and their energy is: $\mathrm{E}_{0}(1+\mathrm{h})$ and the sum of nodes present in each cluster: $\mathrm{N}_{\mathrm{c}} \mathrm{ls}$. The updating energy of each cluster is calculated by the following equation (v).

$$
\mathbf{E}_{\mathrm{cl}}=\mathbf{N}_{\mathrm{cl}} \mathbf{E}_{0}(\mathbf{l}+\mathbf{h j})
$$


The sum of initial energy of an entire cluster is provided by equation - (vi).

$$
\mathbf{E}_{\mathrm{tot}}=\sum_{\mathrm{cl}=1}^{\mathrm{n}} \mathrm{Ecl}
$$

Where n:-sum of cluster nodes.

\section{OUR Proposed Method}

This research work calculates performance issues of energy efficient wireless routing protocols using divide and conquer based quad tree technology. The wsns are very popular name in heterogeneous networks. This research work propounds and evaluates DCQMS-Leach an energy efficient routing protocol in wsns. The technique of divide and conquer based quad tree technology divides the wsn region consisting of sensor nodes into four static cluster regions and then each cluster isolates DCQMS-Leach protocol. The newly proposed DCQMS-Leach routing protocol is derived from the original leach protocol. In this research work we have chosen the cluster head of various dissimilar clusters based on Distributed Energy Efficient Clustering (DEEC) protocol which allows dissimilar nodes to cope with the heterogeneous nature of wsn. Due to small static cluster hops each hop decreases its broadcast message power because of limited static coverage area. This research work calculates performance issues of DCQMS-Leach protocol using Mat lab. The simulation result shows that DCQMS-Leach is better than Leach, Ad-leach, and Deec wireless energy efficient routing protocols. In the leach protocol each node picks its cluster head individually. Therefore the first cluster head (ch) chosen methodology is initiated. After picking of each cluster head the cluster head formation phase is automatically created. When all nodes are assigned themselves to an appropriate cluster each cluster head allocates a time slot to its client hop. All hops use their allocated time slot to communicate with their respective ch.

The leach protocol uses stochastic ch selection algorithm for homogeneous networks. The ch is a hop which collects data from all of its clients, hops, compresses it and then transmits it towards bs. During this entire period, ch consumes extra energy. The node which is selected as a ch dies out quickly due to extra energy burden. In leach protocol, all hops share this load. To divide this additional load uniformly for all hops leach proposes a new technique where by each hop $\mathrm{H}_{\mathrm{i}}=(1,2,3, \ldots \ldots \ldots \mathrm{N})$ becomes cluster head (ch) after 1/p rounds. However as the network evolves the energy level of all nodes differ due to different traffic load and their distance from bs, which leads to unfair cluster head (uch) retrieval and nodes with low energy level die out.

\subsection{Divide And Conquer Quad Tree (DCQMS) BaSed Static Multi-Hop Leach Protocol RePresentation In Wireless Sensor NeTWORK.}

Let us assume that whenever any eewsn which consists of $n$ number of nodes, first we have to divide $\mathrm{n}$ number of nodes of wsns by using quad tree concept. A quad tree is a data structure in which each internal node has exactly four children. In a quad tree concept, each node specifies a rectangle (bounding box) which covers some part of the space being indexed with the root node covering an entire area.

The quad tree is a concept which belongs to an analysis and design of algorithms in computer science which splits a rectangular section of wsns into four different quadrants. Ternary tree divides a tree into three siblings such as left sibling, middle sibling and right sibling etc. In a similar manner a quad tree divides a non-linear data structure tree into four siblings namely one left, two middle siblings and one right sibling etc. The fig 2 shows a wsn which consists of different type of sensor nodes with a divide and conquer based quad tree concept. The below fig 3 represents how to divide a wsn into a four quadrants such as q1, q2, q3 and q4. We have deployed a bs (sink) at the centre of all the four quadrants. The bs is one which controls all the ch which belong to each quadrant like q1, q2, q3 and q4. Each quadrant consists of different types of wireless nodes connected to a cluster head (ch). 
If we want to simplify the complex number of wireless nodes in a wsn then divide and conquer quad tree based multi-hop leach protocol can be considered thus as a best solution. The quad tree based static leach eewsn is a manually simulated routing protocol which is implemented by using Mat lab. The cluster nodes (cns) always transmit data to the bs. The location of bs lies in the centre of all the four quadrants. The quad tree based static leach protocol supports recursion process to split each quadrant into equivalent four quadrants. The recursion split function is called infinite number of times to divide a rectangle of eewsn into infinite number of small quadrants. This process is exactly similar to the process of splitting an atom into the smallest individual particle. The entire eewsn controls a main bs (sink) and other small bss of each smaller quadrant's and other chs of wsns. The quad tree is one which actually supports only four child nodes such as north west (nw), north east (ne), south west (sw) and south east(se). The nw, ne, sw, se are also called as q1, q2, q3 and q4 quadrants. One of the limitation of quad tree based multi-hop static leach routing protocol is that it exactly support only four quadrants (child nodes) at each different level. The general architecture is shown in fig 2.

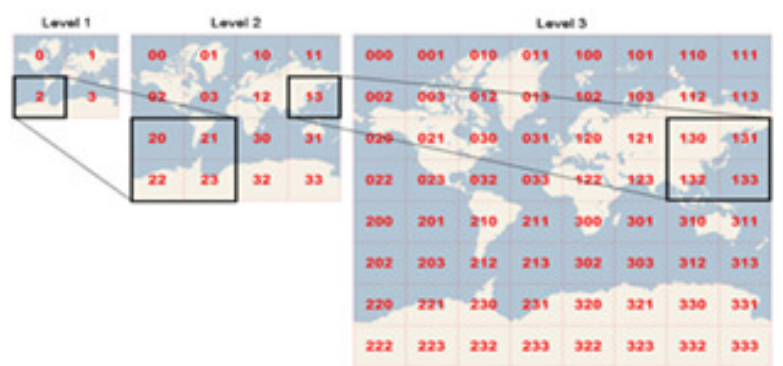

Fig 2.Quad tree data structures using levels.

In wsn the two important criteria to select the chs are as follows:

i) The node which is closer to the base station is chosen as ch.

ii) Energy level of each node which belongs to the wsn.

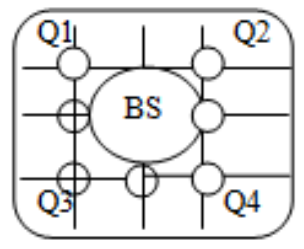

Fig.3.Specification of quad tree based on multi-hop static leach eewsns routing protocol architecture. The fig 4 indicates each quadrant with only four eews nodes in wsns.

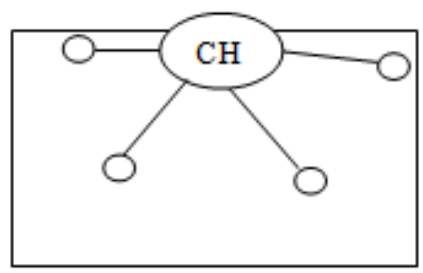

Fig.4.Representation of cluster heads with 4 quadrants. 


\subsection{Mathematical Modeling Of EewSNS}

In this section we are introducing some probabilistic clustering algorithms for eewsns. Let us first narrate the network model which is used in the analysis and simulations. Let us suppose $\mathrm{n}$ sensor nodes are scattered in an area of $\mathrm{M}$ x M square meters and a base station is installed at the centre of sensing area. The deployment techniques of sensor nodes are broadly partitioned into two types namely Hand (sensor nodes are uniformly distributed to a district) and random way (nodes are stochastically installed to a specific jurisdiction). Let us consider that the least initial energy of all the nodes is $H_{0}$. When each node $S_{j}$ have $\alpha_{j}$ times more initial energy i.e. $H_{0}\left(1+\alpha_{j}\right)$, the total energy of whole network is indicated by

$$
\mathbf{H}_{\mathbf{t}}=\mathbf{T}_{\text {otal }}=\sum_{i=1}^{n} \mathrm{Ho}(1+\alpha \mathbf{j}) \text {. }
$$

If $\alpha_{\mathrm{j}}=0$ for all nodes which corresponds to the case of homogeneous networks. In case of two level heterogeneous networks $\alpha_{\mathrm{j}}$ has two values. In case of three levels of heterogeneous networks $\alpha_{j}$ has three values where as in case of different $\alpha_{j}$ values it supports for multilevel heterogeneous wsns.

\subsection{Threshold Computation Of Leach Protocol}

Leach is the most popular clustering protocol based on probability of homogeneous wireless sensor networks. In leach every node is equipped with same initial energy and it contains the same probability $\mathrm{H}_{\mathrm{opt}}$ to become cluster head. Though leach does not consider energy consumption yet it introduces a threshold $\mathrm{T}_{\mathrm{s}}$ to rotate the role of cluster head in order to equally distribute energy consumption through the whole network.

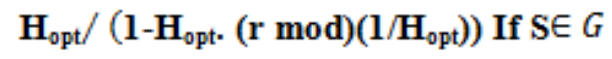

Threshold $\left(T_{s}\right)=0$

(viii)

\section{Otherwise}

Where $\mathrm{H}_{\mathrm{opt}}$ is the desired percentage of cluster heads (chs) for ex: $\mathrm{H}_{\mathrm{opt}}=0.05, \mathrm{r}$ is a current round and $\mathrm{G}$ is the sequence of nodes which are not picked as cluster heads in the previous $1 / \mathrm{p}$ rounds. Each node in the current set $\mathrm{G}$ selects a random number from 0 to 1 . When this number is lesser than threshold $\mathrm{T}_{\mathrm{s}}$ it becomes a cluster head. Due to the introduction of $\mathrm{T}_{\mathrm{s}}$ the main role of cluster head is rotated between every two rounds. Mean while an optimal number of cluster heads achieved in every round of leach is calculated by the following formula.

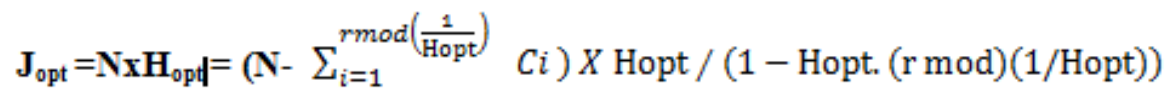

According to the literature the best $\mathrm{J}_{\mathrm{opt}}$ is derived by

$$
\begin{aligned}
& \mathbf{J}_{\mathrm{opt}}=\sqrt{\mathbf{N}} \sqrt{\varepsilon_{\mathrm{fs}}} \mathbf{M} \\
& \sqrt{\mathbf{2} \Pi \sqrt{\varepsilon_{\mathrm{mD}}} \mathbf{d}^{2}{ }_{\mathrm{toBs}}}
\end{aligned}
$$

Where $\varepsilon_{\mathrm{fs}}$ and $\varepsilon_{\mathrm{mp}}$ are two important parameters of radio energy dissipation model. $\mathrm{d}_{\mathrm{toBS}}^{2}$ is an average distance between a ch and a bs. 


\subsection{Probability Computation Of Heterogeneous normal And Advanced NODES.}

In DCQMS-leach clustering routing protocol, the nodes are broadly categorized into two types according to the amount of an initial energy. The first type of node is normal node with little energy and the second type of node is an advanced node, with $\alpha$ times more energy than a normal node. Their selecting probabilities are as follows.

$$
\begin{aligned}
& \mathbf{P}_{\mathrm{urm}}=\mathbf{H}_{\mathrm{opt}} \frac{\mathbf{X E _ { \mathrm { urm } }}}{\mathbf{E}_{\mathrm{t}}}=\frac{\mathbf{H}_{0 \mathrm{pt}} \mathbf{X} \mathbf{N E}_{0}}{\mathbf{N}(1-\mathrm{m}) \mathbf{E}_{0}+\mathbf{N m E}_{0}(1+\boldsymbol{\alpha})} \\
& \mathbf{H}_{\mathbf{0 p t}} / \mathbf{l}+\mathbf{m} . \boldsymbol{\alpha} \\
& \mathbf{P}_{\mathrm{adv}}=\underset{\mathbf{E}}{\mathbf{H}_{0 \mathrm{pt}} \mathbf{X} \mathbf{E}_{\mathrm{adv}}} \frac{=}{\mathbf{N}(1-\mathbf{m})} \mathbf{H}_{\mathrm{opt}} \frac{\mathbf{X} \mathbf{N E}_{0}(1+\alpha)}{\mathbf{E}_{0}+\mathbf{N m E}_{0}(1+\alpha)} \\
& =\mathbf{H}_{\mathrm{opt}} \cdot(1+\alpha) / 1+\mathbf{m} \cdot \boldsymbol{\alpha}
\end{aligned}
$$

Where $\mathrm{m}$ is the fraction of advanced nodes, $\mathrm{H}_{\mathrm{opt}}$ is the optimal clustering probability for corresponding homogeneous wsns. By comparing equations (11) and (12) we can confirm that normal nodes are assigned smaller probability while advanced nodes are accorded higher probability. In DEEC algorithm, cluster head selection is based on residual energy rather than an initial energy of DCQMS-Leach. When the probability is dynamically changing, the residual energy of every node rapidly decreases as the time passes. The probability $P_{i}$ for node $S_{i}$ is given as

$$
\begin{aligned}
& \mathbf{P}_{\mathbf{i}}=\mathbf{H}_{0 \mathrm{pt}} \mathbf{E}_{\mathbf{i}}(\mathbf{r}) / \mathbf{E}_{\mathrm{avg}}(\mathbf{r}) \\
& \mathbf{E}_{\mathrm{avg}}(\mathbf{r})=\mathbf{l} / \mathbf{N} \sum_{i=1}^{N} E i(r) \\
& \mathbf{P}_{\mathbf{i}}=\mathbf{H}_{\mathrm{opt}} \mathbf{E}_{\mathbf{i}}(\mathbf{r}) / \mathbf{E}_{\mathrm{avg}}(\mathbf{r}) \\
& \mathbf{E}_{\mathrm{avg}}(\mathbf{r})=\mathbf{1} / \mathbf{N} \sum_{i=1}^{N} E i(r) \\
& =\mathbf{H}_{\mathbf{0 p t}} \mathbf{E}_{\mathbf{i}}(\mathbf{r}) / \mathbf{E}_{\mathrm{avg}}[\mathbf{r}) \\
& \mathbf{E}_{\mathrm{avg}}(\mathbf{r})=\mathbf{l} / \mathbf{N} \sum_{i=1}^{N} E i(r)
\end{aligned}
$$

Where $E_{i}$ is the residual energy of node $S_{i}$ in round $r$ and $E_{a v g}$ is an average residual energy node in round $\mathrm{r}$. For heterogeneous nodes $\mathrm{H}_{\mathrm{opt}}$ will be replaced by

$$
\mathrm{P}\left(\mathrm{s}_{\mathrm{i}}\right)=\mathbf{H}_{\mathrm{opt}} \cdot \mathrm{NE}\left(\mathrm{S}_{\mathrm{i}}\right) / \mathbf{E}_{\mathrm{t}}
$$




$$
\frac{\mathbf{H}_{0 p t} \mathbf{N E}_{0}\left(\mathbf{1}+\boldsymbol{\alpha}_{\mathrm{i}}\right)}{\mathbf{E}_{0}\left(\mathbf{N}+\sum_{\mathrm{i}=1}^{\mathrm{N}} \alpha \mathrm{i}\right)} \quad=\frac{\mathbf{H}_{\mathrm{ppt}}\left(\mathbf{1}+\boldsymbol{\alpha}_{\mathrm{i}}\right)}{\left(\mathbf{N}+\sum_{\mathrm{i}=1}^{\mathrm{N}} \alpha \mathrm{i}\right)}
$$

The more generalized $\mathrm{P}_{\mathrm{i}}$ can also be expressed by the following equation

$$
\mathbf{P}_{\mathrm{i}}(\mathbf{r})=\frac{\mathbf{H}_{\mathrm{opt}} \mathbf{N}\left(\mathbf{l}+\boldsymbol{\alpha}_{\mathrm{i}}\right) \mathbf{E}_{\mathrm{i}}(\mathbf{r})}{\left(\mathbf{N}+\sum_{\mathrm{i}=1}^{\mathrm{N}} \alpha \mathrm{i}\right) \mathbf{E}_{\mathrm{avg}}}
$$

\subsection{Algorithm of DCQMS - LEACH :}

Input : Energy

Output: Reduce energy consumption and increase lifetime of wsns.

Step-1:Start of an algorithm.

Step-2:Let us assume rectangle(r1)

Step-3:Divide R1 into four quadrants such as q1,q2,q3 and q4.

Step-4:Locate bs at the centre of rectangle.

Location_bs (base station) $=($ Size of rectangle R1/2,Size of rectangle R1/2)

Step-5:Let us consider ch1 with n number of nodes using multi hop concept.

Step-6:Recursively sub divide quadrant into four sub quadrants $\mathrm{q} 1^{1}, \mathrm{q} 1^{11}, \mathrm{q} 1^{111}$ and $\mathrm{q}^{1111}$ until a sub quadrant has only one point in it.

a:Each division results in a single node with four sub quadrants.

Step-7:Select ch as a root node with 'n' number of nodes and each ch connect with four quadrant and sub quadrant chs.

Step-8:Forward the data from n number of nodes to the each quadrant and sub quadrant chs. The chs aggregate data from all $n$ number of nodes in each quadrant and sub quadrant and finally the whole data is forwarded to the bs.

Step-9:End of an algorithm.

\section{Simulations AND Discussions}

This research work was conducted as an innovative experiment to study the mathematical model based on DCQMS-Leach protocol for an eewsns. An extensive simulations results were conducted by using Mat lab simulation environment to compute the implementation, evaluation and performance issues of DCQMS-Leach with DEEC and advanced leach protocol for generating eewsns. The simulation result shows that DCQMS-Leach extends the network life time, increases the overall throughput, reduces energy consumption and optimizes the number of cluster heads. This research work compares DCQMS-Leach heterogeneous protocol with DEEC protocol on the basis of energy dissipation and longevity of network [37,38,39]. The various performance metrics for simulation purpose to simulate energy efficient heterogeneous static routing protocol are:

i. The number of dead nodes for each round.

ii. The Stability Period (SP) is defined as the period starts from the network operation and ends between the first dead node.

iii. The Instability Period (ISP) (Unstable Jurisdiction) (UJ) is the period that exists between the first dead node and the last dead node.

iv. The number of alive nodes/round.

v. The number of dead nodes/round.

vi. Throughput=Number of packets sent from ch (source) to the bs (Destination). 
vii. Network Life Time=An interval exists between beginning of network operation and the death of last sensor node.

viii. Reliability:Reliability depends on the measurement of Stable Jurisdiction (SJ) and Unstable Jurisdiction (UJ). When SJ is larger and UJ is smaller the reliability.

ix. The number of cluster heads/round:The total number of nodes chosen as chs from the whole wsn in each round. The chs are usually responsible for accepting data from the member node and send all data after aggregation to the sink hop.

Table-1: Simulation Parameter Settings.

\begin{tabular}{|c|c|c|}
\hline Sl.No & Parameters & Corresponding values \\
\hline 1 & $\mathrm{~N}$ & 300 \\
\hline 2 & A & 1 \\
\hline 3 & $\mathrm{M}$ & 0.3 \\
\hline 4 & $\mathrm{H}_{\text {opt }}$ (Optimal probability) & 0.1 \\
\hline 5 & $\mathrm{~K}$ (Packet size) & 4000 \\
\hline 6 & $\mathrm{E}_{0}$ (Energy for normal nodes) & $0.5 \mathrm{~J}$ \\
\hline 7 & $\begin{array}{lr}\text { Eelect(Radio } & \text { electronics } \\
\text { energy)(Transmitter/receiver electronics) }\end{array}$ & $50 \mathrm{~nJ} / \mathrm{bit}$ \\
\hline 8 & EDA (Data aggregation energy cost). & $5 \mathrm{~nJ} / \mathrm{bit} / \mathrm{message}$ \\
\hline 9 & $\varepsilon f s$ (Transmit amplifier) if dmax to $\mathrm{BS} \ll=\mathrm{d}_{0}$ & $10 \mathrm{pJ} / \mathrm{bit} / \mathrm{m}^{2}$ \\
\hline 10 & $\operatorname{smp}$ (Transmit amplifier) if $f_{d \max }$ to $\mathrm{BS}>=\mathrm{d}_{0}$ & $0.0013 \mathrm{pJ} / \mathrm{bit} / \mathrm{m}^{4}$ \\
\hline 11 & Network size & $500 \mathrm{~m} \times 500 \mathrm{~m}$ \\
\hline 12 & $\begin{array}{l}\mathrm{b} \text { (Proportion of intermediate nodes with } \\
\mu \text { times more energy than normal nodes) }\end{array}$ & 0.3 \\
\hline
\end{tabular}

The Performance of DCQMS-Leach protocol is efficiently scrutinized in this section using Mat lab. A heterogeneous wsn which contains $n=100$ nodes are first considered in this simulation. The value of $\mathrm{m}=0.3$ which specifies there are $30 \%$ advanced hops containing a times more energy than the normal nodes. The simulation domain is specified with $\mathrm{x}=500 \mathrm{~m} \times \mathrm{y}=500 \mathrm{~m}$ which represents a rectangular field. The Table-1 shows the simulation parameters required to simulate the DCQMS-Leach protocol in eewsns. In the second phase this research work compares the DCQMS-Leach energy efficient routing protocols in eewsns. The below figs 5,6,7 and 8 show the comparison of number of dead hops, number of alive hops, sum of datagrams sent to the bs and the total number of chs.
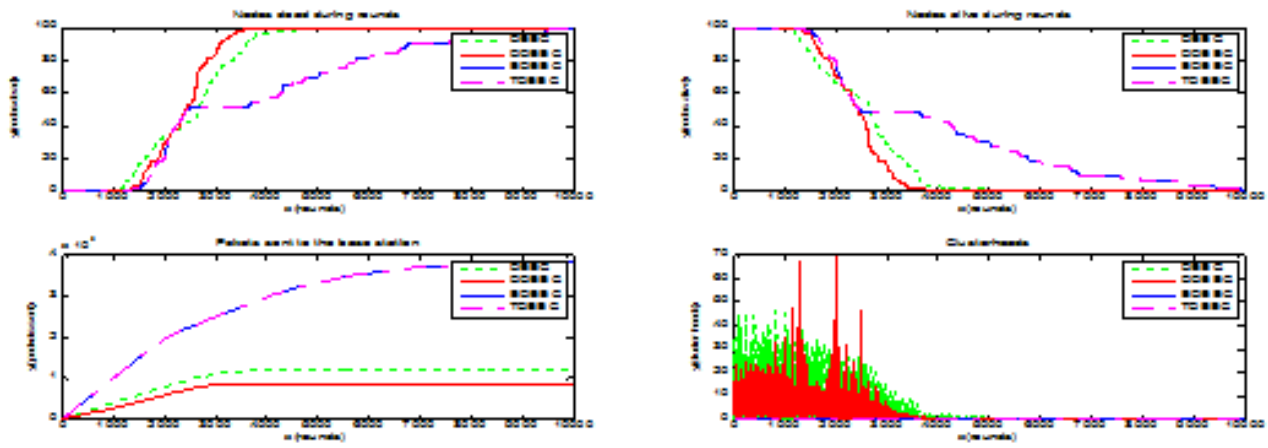

Fig.5,6,7,8:Comparison of DEEC,DDEEC,EDEEC and TDEEC energy efficient routing protocols for alive nodes, dead nodes, packets sent to the bs, chs. 
In the above fig 5, 6, 7 and 8 the research work comparing the DEEC, DDEEC, EDEEC and TDEEC energy efficient routing protocols. The fig 5 specifies the number of dead nodes with respect to number of rounds in DEEC, DDEEC, EDEEC and TDEEC the research work proves that TDEEC and EDEEC supports for maximum number of dead nodes with respect to number of rounds where as DEEC and DDEEC cooperates for minimum number of dead nodes with respect to number of rounds. In fig 6 the research work has taken an opportunity to compare number of alive nodes with respect to number of rounds and the research work proves that DEEC energy efficient routing protocol supports minimum number of alive nodes as compared to the DDEEC, EDEEC and TDEEC where as TDEEC, EDEEC and DDEEC are supporting for maximum number of alive nodes with respect to number of rounds.

Therefore the research work confirms that TDEEC energy efficient routing protocol is better than EDEEC, DDEEC and DEEC energy efficient routing protocols. In the fig 7 this research work compares the TDEEC energy efficient routing protocol with the other three different kinds of energy efficient routing protocols in terms of number of packets sent to the sink with respect to number of rounds. The calculated research results confirm that TDEEC is sending maximum number of packets as compared to other three different categories of protocols. Therefore TDEEC is performing better than EDEEC, DDEEC and DEEC. In fig 8 this research work also computes DEEC, DDEEC, EDEEC and TDEEC energy efficient routing protocols by comparing chs with number of rounds. The DDEEC energy efficient routing protocol has more number of cluster head with respect to number of rounds. Therefore this research work also confirms that the DDEEC performance is better than EDEEC, TDEEC and DEEC energy efficient routing protocols.

The fig 9 shows the comparison and contrast between the energy of the proposed DCQMS -Leach protocol with DEEC energy efficient routing protocol by using bar charts.

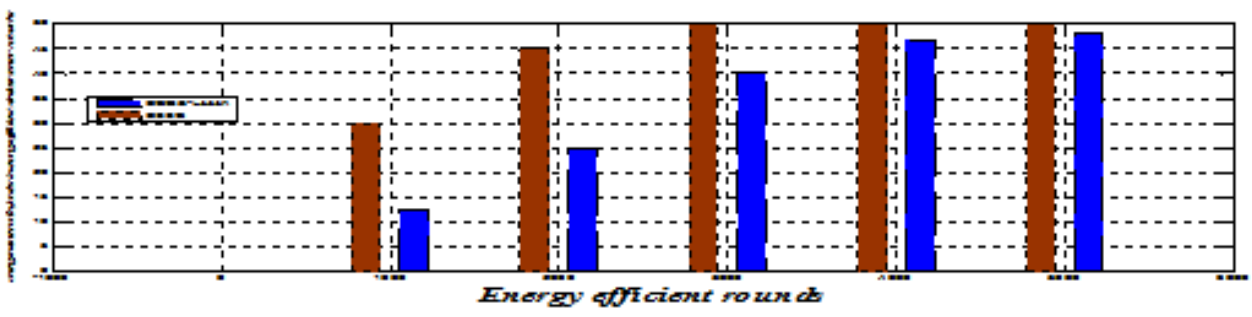

Fig 9. Energy comparison between DCQS -Leach and DEEC energy efficient routing protocol when $\mathrm{m}=0.3$ and $\mathrm{a}=1$.

The fig 9 shows the results of energy utilized between DCQMS-Leach and DEEC routing protocol with respect to round. The proposed DCQMS - Leach protocol is better than DEEC and Leach protocol threshold is around 4000 rounds when $m=0.3$ and $a=1$. The energy consumption of Leach, DEEC and DCQMS-Leach is shown in fig 10.

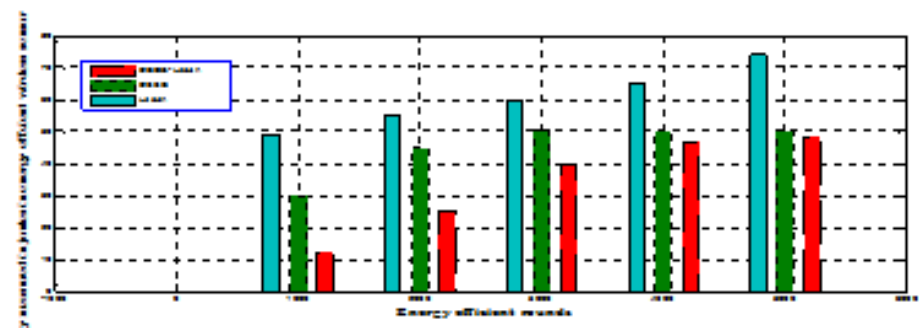

Fig 10. Energy consumption in Leach, DEEC and DCQMS-Leach protocol in eewsns. 
The fig 11 shows the prediction of throughput when $\mathrm{m}=0.3$ and $\mathrm{a}=1$.

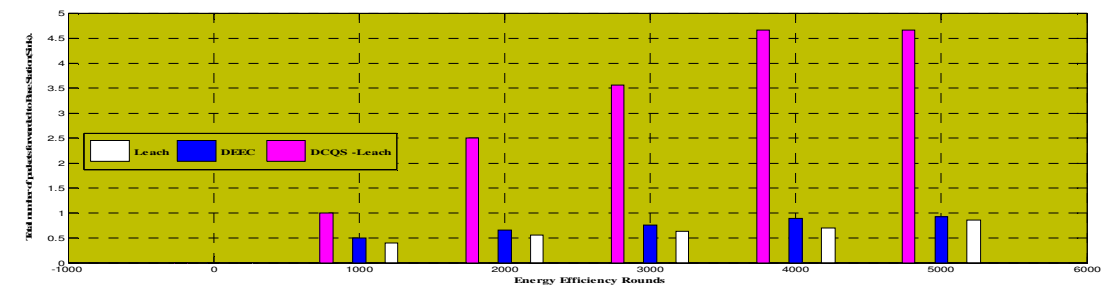

Fig 11.Comparison of throughput of Leach, DEEC and DCQMS-Leach routing protocols.

The fig 12 shows the total number of alive and dead nodes in an energy efficient routing protocol with respect to number of different rounds.

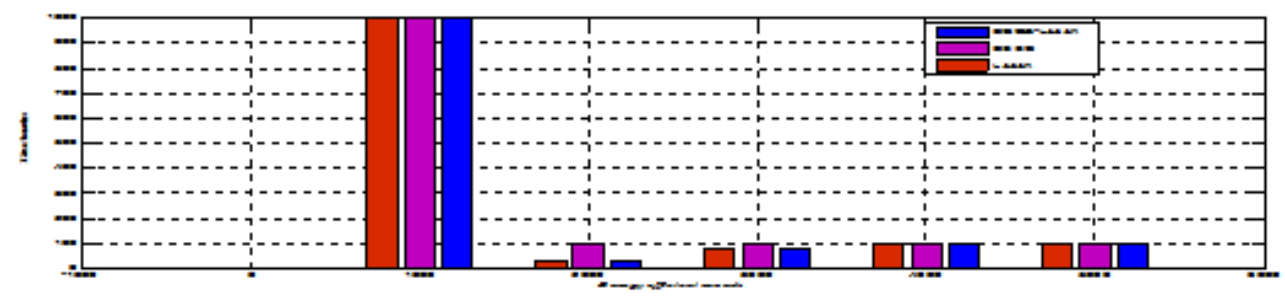

Fig 12.The total number of dead nodes in Leach, DEEC and DCQMS-Leach routing protocols.

\section{EPILOGUE}

This research work has proposed a novel DCQMS-Leach protocol to save energy consumption in eewsns. This research work was compared the newly proposed DCQMS-Leach routing protocol with previous DEEC and leach energy efficient routing protocol in wsns. In this proposed scheme we have considered a divide and conquer quad tree based graph theory concept in order to verify and validate the comparison of energy consumption, dead nodes vs. alive nodes, and throughput with respect to the number of rounds. This research work easily prove and confirm that DCQMSLeach is a suitable energy efficient routing protocol to save energy in a heterogeneous wsns. In future the research work shown in this paper could be extended in many folds. Indeed to the best of our knowledge this is the first attempt towards the representation computing and reducing energy efficiency of wireless sensor node in wsns by the use of

i. Divide and conquer strategy

ii. Quad tree concept.

iii.

In future this research work can also be extended with

i. List ranking based multi hop energy efficient routing protocol for wsns

ii. Double linked list, single linked list, reverse single, double linked list etc.

iii. Greedy based, back tracking based multi hop energy efficient routing protocol.

iv. Slice wise curtail based energy efficient protocol for single, multi hop routing.

\section{ACKNOWLEDGEMENTS}

The second author is thankful and grateful to University of Mysuru (UoM) and Visveshwaraiah Technological University (VTU) for providing me an opportunity to work on Quad Tree Based Static Multi hop Leach Energy Efficient Routing Protocol: A Novel Approach. The second and first authors also thank all the anonymous reviewers who gave valuable suggestions and comments to rectify mistakes done while writing this research paper. This work is a part of VTU research work of Abdulmalek Maresh Hasan Ali of Yemen. 
International Journal of Computer Networks \& Communications (IJCNC) Vol.10, No.1, January 2018

\section{REFERENCES}

[1] Donald E.Knuth(1998), The art of Computer Programming:Volume-3,Sorting and Searching,Second edition,Addison-Wesley.

[2] Thomas H.Cormen,Charles E.Leiserson and Ronald L.Rivest(2000),Introduction to Algorithms,MIT Press.

[3] Brassard.G.and Bratley.P.(1996),Fundamental of Algorithmics,Prentice-Hall.

[4] Anany V.Levitin(2002),Introduction to the Design and Analysis of Algorithms,Addison-Wesley.

[5] K.Sha,J.Gehlot and R.Greve(2012),"Multipath routing techniques in Wireless Sensor Networks:A Survey",Wireless Peers Communications, Springer.

[6] W.R.Heinzelman,A.P.Chandrakasan and H.Balakrishnan(2002),"An Application-Specific Protocol Architecture for Wireless Micro Sensor Networks",IEEE Transactions on Wireless Communications, 1(4),pp 660-670.

[7] J.Hanumanthappa(2015), "An Investigations Into The Design,Performance And Evaluation Of A Novel Energy Efficient Multipath Routing Algorithms in Wireless Sensor Networks",in Proceedings of the National Conference on Computer Science and its Applications.

[8] Radi Marjan,Dezfouli Behnam,Bakar Kamalrulnizam Abu,S.Abd Razak and M.A.Nematbaksh(2011),'Interference Aware Multipath Routing Protocol for QoS improvement in event driven wireless sensor networks",Tsinghua Sci.Tech,vol.16,no.5,pp 475-490.

[9] Wendi Rabiner Heinzelman,Joanna Kulik and Hari Balakrishnan(1999),"Adaptive protocols for Information dissemination in Wireless Sensor Networks",In Proceedings of the 5th Annual ACM/IEEE International Conference on Mobile Computing and Networking,Mobicom,pp 174-185.

[10] J.N.Al-karaki and A.E.Kamal(2004),"Routing Techniques in Wireless Sensor Networks:A Survey",IEEE Wireless Communications, 11(6):6-28.

[11] W.Heinzelman,A.Chandrakasan,H.Balakrishnan(2000),"An Energy Efficient Communication Protocol for Wireless Micro sensor Networks",in Proceedings of the 33rd Hawaiian International Conference on System Sciences,pp 1-10.

[12] Y.Jin,L.Wang,Y.Kim and X.Yang,EEMC(2005),"An Energy Efficient Clustering Scheme in Wireless Sensor Networks",in Proceedings of the IEEE International Performance Computing and Communications Conference (IPCCC),pp 535-540.

[13] J.Broch,D.Maltz,D.Johnson,Y.Hu and J.Jetcheva(1998),"A Performance Comparison of Multi Hop Wireless Ad-hoc Routing Protocols".In Proc of 4th ACM International Conference on Mobile Computing and Networking(Mobicom).

[14] H.Zimmermann(1980),’OSI Reference Model-The ISO Model of Architecture for Open Systems Inter Connection",IEEE Transactions on Communications, 28(4).

[15] J.Hanumanthappa(2015),"Comparison and Contrast between the Performance issues of T-SEP and ESEP in Energy Efficient Wireless Sensor Networks (EFWSN's)",in proceedings of the UGC Sponsored State Level Conference on Next-Gen Computing (NGC-2015):Challenges and Opportunities,Post Graduate Department of Computer Science,SBRR MahaJana First Grade College,pp 89-101.

[16] J.Hanumanthappa(2015),'Performance and Evaluation of an Intra Cluster and Inter Cluster Novel Graph Theoretic Routing Algorithm (GTRA) in MANET's",in proceedings of the UGC Sponsored State Level Conference on Next-Gen Computing (NGC):Challenges and Opportunities,Post Graduate Department of Computer Science,SBRR MahaJana First Grade College,pp 109-115.

[17] J.Hanumanthappa(2015),'Mathematical Modeling of K-CCP Protocol of Coverage and Connectivity in wireless sensor networks",in Proceedings of the National Conference on Computer Science and its Applications (NCCSA),Dept of CS,Shri Shiradi Saibaba College of Engineering,Bengaluru.

[18] J.Hanumanthappa,Abdulmalek Maresh Hasan Ali(2017),"Node multi homing based policy routing using Mobile IPv6 in ns2",in proceedings of the national conference on advanced information technology (NCAIT),SJBIT,Bengaluru,pp 169-175.

[19] J.Hanumanthappa,Abdulmalek Maresh Hasan Ali(2017),"'Comparison and Contrast between the performance issues of $\mathrm{mDBR}$ and $\mathrm{mCoDBR}$ novel cooperative routing protocols in under water sensor networks (UWSN's)",in International Journal Of Research Science,Engineering Technology (IJRSET),pp 21-28. 
International Journal of Computer Networks \& Communications (IJCNC) Vol.10, No.1, January 2018

[20] J.Hanumanthappa(2015),"Investigations Into The Design,Performance And Evaluation Of A Novel Energy Efficient Multipath Routing Algorithms in Wireless Sensor Networks",in Proceedings of National Conference on Computer Science and its Applications (NCCSA),Dept of CS,Shri Shiradi Saibaba College of Engineering,Bengaluru.

[21] I.F.Akyildiz,W.Su,Y.Sankarasubramaniam and E.Cayirci(2002),"A Survey on Sensor Networks",IEEE Communication Magazine,pp 102-114.

[22] A.Chakraborty,R.Rout,,A.Chakrabarti and S.Ghosh(2013),"On Network Lifetime expectancy with realistic sensing and traffic generation model in Wireless Sensor Networks", IEEE Sensors Journal,Vol.13,no.7,pp 2771-2779.

[23] J.Hanumanthappa,Ahmed Weishel A.Alfarjat,Prof.Sheshadri.H.S(2017),"Mathematical Modeling of Security Issues of WLAN's using Space Time Processing in DSP”,International Journal of Computer Science and Information Security (IJCSIS), Vol.15.No.8,pp 320-330.

[24] J.Hanumanthappa,AbdulMalek Maresh Hassan Ali(2017), Quad Tree Based Static Multi Hop Leach Energy Efficient Routing Protocol:A Novel Graph Theoretic approach, InternationalJournal of Computer Network and Communications(IJCNC), Vol.10.No.8,pp 1-21.

[25] Georgios Smaragdakis,Ibrahim Matta,Azer Bestavros(2002),"SEP:A Stable Election Protocol for clustered heterogeneous wireless sensor networks",Technical Report BUCS-TR-2004-022.

[26] E.J.Duerte-Melo,M.Liu(2002),"Analysis of energy consumption and life time of heterogeneous wireless sensor networks",In Proceedings of the IEEE Global Tele Communication Conference (Globe Com),pp 21-25.

[27] V.Mhatre and C.Rosenberg(2004),"Homogeneous vs Heterogeneous Clustered SensorNetworks:A Comparative Study",In Proceedings of the IEEE International Conference on Communications (ICC).

[28] Hanan Samet,"A Top-Down Quadtree traversal algorithm",IEEE Transaction on Pattern Analysis and Machine Intelligence,Vol,PAMI-7,No.1,January, 1985.

[29] Hanan Samet,"Distance Transform for Images represented by Quadtrees,'IEEE Transactions on Pattern Analysis and Machine Intelligence,Vol,PAMI-4,No.3.,May,1982.

[30] David M.Mark,David J.Abel,'Linear Quadtrees from Vector representations of Polygons",IEEE Transactions on Pattern Analysis and Machine Intelligence,Vol,PAMI-7,No.3.,May,1985.

[31] Jeffrey Wildman,Steven Weber,'On Protocol and Physical Interference Models in Poisson Wireless Networks,IEEE Transactions on Wireless Communications,2017,Issue:99,Volume:PP,pp 1-1.

[32] Ali Bayat,Sonia Aissa,'Full-Duplex Cognitive Radio with Asynchronous Energy Efficient Sensing”,IEEE Transactions on Wireless Communications,Volume:PP,Issue:99,2017,pp 1-1.

[33] Stephanie Lindsey,Cauligi Raghavendra,'Data Gathering Algorithms in Sensor Networks energy metrics using,IEEE Transactions on Parallel and Distributed Systems,Vol.13.,No.9., September 2002.

[34] T.Rapport,Wireless Communications:Principles and Practice,Prentice-Hall Inc,New Jersey, 1996.

[35] J.Broch,D.Maltz,D.Johnson,"A Performance comparison of Multi-Hop Wireless Ad-hoc Network routing protocols,In Proc 4th ACM international conference on mobile computing and Networking(Mobicom'98), October 1998.

[36] Nadeem Q et al(2013) "M Gear-Gateway based energy aware multihop-routing protocol for WSN's". In 2013 8th International Conference on Broadband and Wireless Computing Communication applications(BWCCA),IEEE.doi.10.1109/BWCCA.2013.35.

[37] J.Hanumanthappa,"An Innovative Simulation,Comparison Methodology \& Framework for evaluating the Performance evaluation metrics of a Novel IPv4/IPv6 Transition Mechanisms: BD-SIIT vs.DSTM,in proceedings of the IEEE First International Conference on Integrated Intelligent Computing(ICIIC-2016),SJBIT,Bengaluru,pp 258-263.

[38] J.Hanumanthappa(2011),A Simulation study on the performance of divide- and-conquer based IPv6 Address LPR in BD-SIIT IPv4/IPv6 transition using a Novel Reduced Segment Table(RST) algorithm in BD-SIIT Translator,Proceedings of International Conference on Computer's and Computing(ICCC'11),Lanzarote,Canary Islands,Spain(ISBN:978-1-61804- 0008).

[39] J.Hanumanthappa,2009,An Overview of Study on Smooth Porting process scenario during IPv6 Transition (TIPv6),in Proceedings of IEEE IACC-09,Patiala,Punjab,INDIA-6-7,March- 2009,pp 2217-2222.

[40] J.Hanumanthappa(2014),’DW\&C:Dollops Wise Curtail IPv4/IPv6 Transition Mechanism Using NS2",InterNational Journal Of Engineering Trends and Technology (IJETT),Vol.14,No.6.,pp 271279. 
[41] Anindita ray,Debashis De,"energy efficient clustering protocol based on K-means(EECPK-means) midpoint algorithm for enhanced network lifetime in wireless sensor networks",IETWireless Sensor Systems,PP 1-11.

[42] J.Hanumanthappa,"IPv6 and IPv4 Threat reviews with Automatic Tunneling and Configuration Tunneling Considerations Transitional Model:-A Case Study for University of Mysore Network ”,International Journal of Computer Science and Information Security,vol.3.,No.1.,2009,pp 1-12.

\section{AuTHORS}

Dr.Hanumanthappa.J .received his BE degree in Computer Science and Engineering at Kuvempu University,Shankarghatta,Karnataka,India in 1998 and his M.Tech Degree in Co mputer Science and Engineering from the National Institute of Technology,Surathkal,Karnataka,India in 2003 and $\mathrm{Ph}$. D in Computer Science and Technology from Mangalore University,Mangalagangothri,Mangalore in 2014. Presently

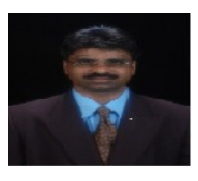
he is working as Assistant Professor (Reader) (Stage-2) at DoS in CS,University of Mysuru,Manasa gangothri,Mysuru-6 since 04/06/2004. He has presented and published more than 50 refereed research papers in IEEE Transaction Journals/IEEE International Springer Journals/ Science Direct Journals, Elsevier Journals. He has also published his highly repetitive research articles in both National and International Conferences. He is also working as a Life Member of Institute of Engineer's, M.I.S.T.E.,CSI,Senior Life Member of IACSIT,IAENG,IEEE,IPv6 forum India etc. He has also reviewed more than 200 research papers for refereed IEEE/ACM/Science Direct/Elsevier/Springer journals and conferences. He has also chaired several paper presentation sessions in International/National Conferences. He is Visiting Faculty of Huang Huai University,Zhumadian,Henan,People Republic of China. His area of research his "Performance issues of Protocols",Wired/Wireless Sensor Networks,Clustering Algorithms etc. Currently he has around 18 Years of Teaching Experience covering highly reputed engineering colleges in Karnataka and DoS in CS,University of Mysuru.

Mr.Abdulmalek Maresh Hasan ali is presently working as a Research Scholar in Visveswaraya Technological University(VTU),Belgaum,Karnataka,India. He passed his Bachelor of Engineering(BE) in Computer Programming in the year 2007 from Al_Yemenia University,Sanaa,Yemen,and his Master's Degree in Master of Technology (M.Tech) in Computer Science and Technology in 2015 from the University of Mysuru.

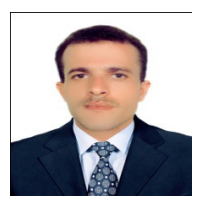
He has presented/published two research papers in reputed national conferences and International Journals. His area of Research Interest his Wireless Sensor Networks, a Clustering algorithms,Performance issues of Protocols, Wired networking etc. 